\title{
Determinación cuantitativa de ésteres grasos del 3-fenilamino- 1,2-propanodiol por espectrofotometría de absorción.
}

\author{
Por R. Gómez Gómez* y A. Vázquez Roncero** \\ * Departamento de Química. Universidad de Castilla-La Mancha. \\ Escuela de Ingeniería Técnica Agrícola de Ciudad Real. \\ ** Instituto de la Grasa y sus Derivados (C. S. I. C.) de Sevilla.
}

RESUMEN

Determinación cuantitativa de ésteres grasos del 3-fenilamino-1,2-propanodiol por espectrofotometria de absorción.

Se ha puesto a punto un método analítico para la determinación cuantitativa de los acilderivados (mono- y di-) del 3fenilamino-1,2-propanodiol. El método consiste en determinar la absorbancia de los complejos coloreados que forman los acilderivados con p-DMACA (reactivo específico de aminas aromáticas primarias y secuandarias). Los límites de confianza calculados a partir de los datos correspondientes a las rectas de calibrado son de $\pm 0,031$ unidades de absorbancia a $500 \mathrm{~nm}$ para el monoacilderivado y $\pm 0,020$ a $510 \mathrm{~nm}$ para el diacilderivado.

La principal aplicación de éste método es proporcionar un instrumento válido para el cálculo del rendimiento de la síntesis de estos productos, además de proporcionar un sistema de análisis fácilmente extensible a otros productos de estructura similar.

PALABRAS-CLAVE: Espectrofotometria de absorción - Ester graso de 3-fenilamino-1,2-propanodiol (análisis) - 3-Fenilamino1,2-propanodiol.

\section{SUMMARY}

Quantitative determination of fatty esters of 3-phenylamine1,2-propanediol by absorption spectrophotometry.

An analytical method for quantitative determination of fatty ester of 3-phenylamine-1,2-propanediol has been developed. The procedure is based on the spectrophotometric determination of coloured compounds formed by reaction of acylderivatives with p-DMACA. Monoacylderivative is quantified at $500 \mathrm{~nm}$ and diacylderivative at $510 \mathrm{~nm}$, with range of $\pm 0,031$ and $\pm 0,020$ absorbance units, respectively.

The essential application of this method is to obtain the yield of synthesis reaction of acylderivatives of 3-phenylamine1,2-propanediol. However, an isolation of every one products by thin layer chromatography is necessary before analytical technical application. Moreover, this procedure supply a way for analysis of similar structure compounds.

KEY-WORDS: Absorption spectrophotometry - Fatty ester of 3-phenylamine-1,2-propanediol (analysis) - 3-Phenylamine-1,2propanediol.

\section{INTRODUCCION.}

El interés por el estudio de los acilderivados de arilaminoalcoholes y más concretamente de los ésteres del 3-fenilamino-1,2-propanodiol se suscitó cuando se encontraron estos productos en bastantes muestras de aceites implicados en el llamado "Síndrome Tóxico" (1). A raiz de la determinación de su estructura, en nuestros laboratorios, hemos creído de interés estudiar su síntesis al tratarse de productos no descritos en la bibliografía (Fig. 1).

El trabajo se ha realizado en dos etapas: en primer lugar la síntesis de los acilderivados por reaccción entre el 3-fenilamino-1,2-propanodiol y los ésteres metílicos de ácidos grasos (2). Esta reacción se ha ensayado también con otros arilaminoalcoholes de estructura muy parecida al 3-fenilamino-1,2-propanodiol con los que se han obtenido resultados satisfactorios (3). $Y$ en segundo lugar, el estudio de las condiciones más adecuadas para obtener estos productos con un rendimiento óptimo.

El primer problema que se nos planteó a la hora de determinar el rendimiento de la reacción fue el disponer de un método analítico idóneo para la cuantificación de la mezcla de reacción. El objeto del presente trabajo es, por lo tanto, el establecimiento del método analítico que nos permita cuantificar estos productos de la forma más directa y rápida posible.

Como ya hemos dicho, la síntesis se lleva a cabo mediante transesterificación de ésteres metílicos de ácidos grasos con 3-fenilamino-1,2-propanodiol en presencia de un catalizador. Por lo tanto, la mezcla de reacción resultante estará compuesta por los acilderivados formados (mono- y di-), además de parte de los reactivos que quedan sin reaccionar. Los primeros ensayos realizados pretendían determinar el rendimiento de la reacción sin necesidad de separar previamente los acilderivados formados. Sin embargo, los resultados obtenidos con diferentes técnicas instrumentales (CG, HPLC) no fueron satisfactorios. 


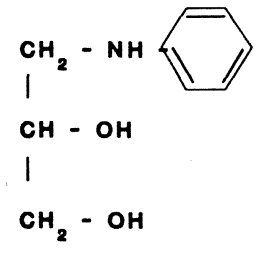

(1)

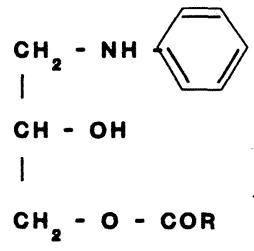

(II)

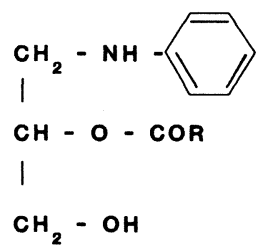

(III)

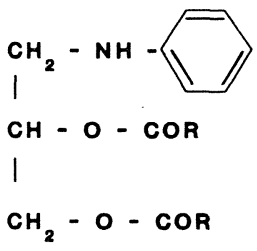

(IV)

Figura 1

Aminoalcohol y sus acilderivados: (I) 3-Fenilamino-1,2-propanodiol; (II) 1-Monoacilderivado; (III) 2-Monoacilderivado; (IV) 1,2-Diacilderivado.

Una vez desechada esta posibilidad se ensayaron varios procedimientos a partir de soluciones purificadas de los acilderivados. El método con el que hemos obtenido mejores resultados y que proponemos en el presente trabajo, se basa en la determinación espectrofotométrica de los complejos coloreados que forman estos productos con $p$-dimetilamino cinamaldehído ( $p$-DMACA). Este reactivo forma unos complejos estables con las aminas aromáticas primarias y secundarias, de color rojo anaranjado a púrpura, que ofrece un camino viable para la cuantificación de estos productos. La especificidad de este reactivo fue establecida por Sakai (4) y en 1982 sirvió de base a Albi y Vioque (5) para la determinación de anilina libre y anilidas grasas en aceites relacionados con el "Síndrome Tóxico".

El procedimiento analítico propuesto consiste en aislar de la mezcla de reacción las cantidades formadas de mono- y diacilderivado mediante cromatografía en capa fina y posteriormente determinar la absorbancia de los complejos coloreados a 500 y 510 $\mathrm{nm}$, respectivamente. La sensibilidad y reproducibilidad de los resultados obtenidos han sido satisfactorios, lo que nos permite evaluar el rendimiento de la reacción con las garantias necesarias.

Hay que tener en cuenta que en las condiciones utilizadas en cromatografía en capa fina (placas de sílice y diclorometano) los dos monoacilderivados isómeros (Fig. 1) no se separan. Por ello, siempre que nos refiramos al monoacilderivado se trata en realidad de la mezcla de ambos isómeros.

\section{PARTE EXPERIMENTAL.}

\section{1. Material y reactivos.}

- Espectrofotómetro UV/Visible Hewlett-Packard Mod. $8450 \mathrm{~A}$, provisto de cubetas de vidrio de $1 \mathrm{~cm}$ de paso.

- Eter etílico, etanol absoluto, diclorometano y ácido clorhídrico de calidad analítica o espectrofotométrica.
- p-Dimetilamino cinamaldehido (p-DMACA), reactivo específico de aminas aromáticas primarias $y$ secundarias. lumna.

- Equipo para cromatografía en capa fina y en co-

- Silicagel 60 GF-254 (Merck) para CCF.

\subsection{Disoluciones patrones.}

Para las puestas a punto del método analítico se han preparado disoluciones patrones del mono- y diacilderivado del 3-fenilamino-1,2-propanodiol. Los productos se sintetizan en el laboratorio mediante alcoholisis de ésteres metílicos de ácidos grasos con el aminoalcohol, utilizando metóxido sódico como catalizador. Posteriormente se purifican mediante cromatografía líquida en columna, sobre gel de sílice y utilizando diclorometano como eluyente (1) (2).

Las disoluciones patrones se obtienen disolviendo los productos puros en éter etílico con unas concentraciones comprendidas entre 10 y 100 ppm.

\subsection{Procedimiento analítico.}

Como hemos dicho anteriormente, el análisis de éstos productos se realiza mediante espectrofotometría de absorción, en el visible, de los complejos coloreados que forman con p-DMACA. El procedimiento seguido para formar dichos complejos es el siguiente:

1.- Se toma $1 \mathrm{ml}$ de la disolución, en éter etílico del producto que se desea medir, se deposita en un matraz aforado de $5 \mathrm{ml}$ y se añaden $2 \mathrm{ml}$ de etanol absoluto.

2.- Se añaden $0,1 \mathrm{ml}$ de una solución de p-DMACA al $1 \%$ en etanol del $95 \%$, con un $5 \%$ de $\mathrm{HCl}$ del $25 \%$ en peso, se agita y se enrasa el matraz con etanol absoluto.

3.- Se esperan 10 min., desde la adición del reactivo, (tiempo necesario para que la intensidad del color desarrollado sea máxima y estable) y se lee la 
absorbancia a la longitud de onda adecuada, según el producto que se desee cuantificar.

Como paso previo se han realizado los espectros de absorción correspondientes a los complejos con pDMACA del 3-fenilamino-1,2-propanodiol y de sus mono- y diacilderivados, con objeto de determinar los máximos de absorción de cada uno de ellos. Estas longitudes de onda serán las utilizadas en la cuantificación de estos productos. Los valores obtenidos son los siguientes:

3-fenilamino-1,2-propanodiol $498 \mathrm{~nm}$

$\begin{array}{ll}\text { Monoacilderivado } & 500 \mathrm{~nm} \\ \text { Diacilderivado } & 510 \mathrm{~nm}\end{array}$

\subsection{Curvas de calibrado.}

Siguiendo el procedimiento analítico descrito se han construido las curvas de calibrado correspondientes al mono- y diacil derivado del 3-fenilamino-1,2propanodiol, a partir de las soluciones patrones de dichos productos (Tabla I). En ambos casos las ecuaciones obtenidas coinciden con la de una recta.

Tabla 1

Curvas de calibrado de los acilderivados del 3-fenilamino-1,2-propanodiol.

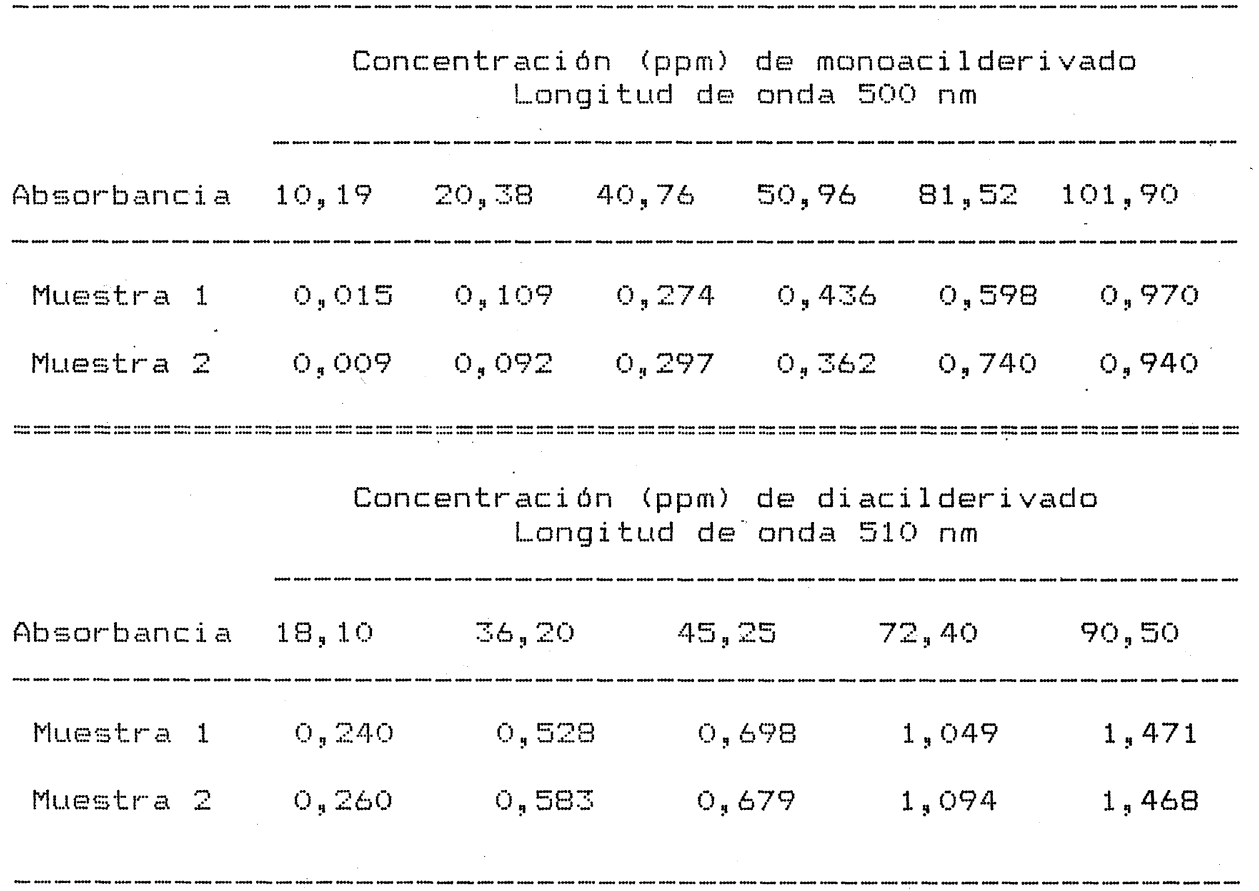

La ecuación obtenida para la recta de calibrado del monoacilderivado es:

$$
C(\mathrm{ppm})=95,92 . A_{500}+10,656 \text { (1) }
$$

con un coeficiente de correlación, $r=0,9986$.

En el caso del diacilderivado, la ecuación de la recta de calibrado es:

$$
C(p p m)=59,22 \cdot A_{510}+3,303
$$

con un coeficiente de correlación, $r=0,9980$.

\subsection{Preparación y análisis de las muestras.}

En el punto 2.3 hemos visto el método propuesto para el análisis del mono- y diacilderivado del 3fenilamino-1,2-propanodiol a partir de los productos puros. Sin embargo, el análisis de estos productos a partir de la mezcla de reacción, nos obliga a realizar una separación previa de ambos debido a que los complejos coloreados que forman, tanto el mono- como el diacilderivado del 3-fenilamino-1,2-propanodiol, absorben a longitudes de onda muy próximas entre sí, por lo que obtendríamos resultados erróneos en la cuantificación de los mismos.

El procedimiento seguido para el análisis es el siguiente:

1.- Se toman alrededor de $50 \mathrm{mg}$ de la mezcla de reacción, exactamente pesados, y se llevan con cloroformo a un matraz aforado de $5 \mathrm{ml}$.

2.- Con una jeringuilla de precisión se toma un volumen determinado (100-200 $\mu \mathrm{l})$ de la solución anterior, se depositan sobre una placa, recubierta con una capa de gel de sílice y se desarrolla utilizando como fase móvil diclorometano. 
3.- Una vez eliminado el disolvente se observa el cromatograma bajo la luz UV de $254 \mathrm{~nm}$, se señalan las bandas correspondientes al mono- $y$ al dialcilderivado del 3-fenilamino-1,2-propanodiol $\left(R_{f} 0,22\right.$ y 0,65 , respectivamente), se raspa la zona correspondiente a cada producto y se eluyen, por separado, con metanol absoluto.

4.- La solución alcohólica se lleva a sequedad a presión reducida y se trasvasa cuantitativamente a un matraz aforado de $2 \mathrm{ml}$, con éter etílico.

5.- Esta solución etérea se utiliza para la determinación espectrofotométrica siguiendo el procedimiento descrito en el punto 2.3.

Las determinaciones se deben realizar siempre por duplicado.

\section{RESULTADOS Y DISCUSION.}

\subsection{Sensibilidad del método analítico.}

Para estimar la sensiblidad del método analítico establecido para la cuantificación de estos productos hemos realizado un estudio estadístico de los datos obtenidos en la construcción de las rectas de calibrado.

El análisis de la varianza realizado con los datos correspondientes el monoacilderivado del 3-fenilamino-1,2-propanodiol revela que lógicamente existen diferencias significativas entre las distintas concentraciones, ya que hemos partido de muestras con concentraciones diferentes. Asímismo se deduce que no existen diferencias significativas entre los valores obtenidos para los replicados de una misma concentración. El valor encontrado para la desviación típica entre replicados es:

$$
s=0,0488
$$

A partir de este valor se han obtenido los Límites de Confianza para una medida dada (11 grados de libertad y una probabilidad del $95 \%$ ) que pueden expresarse como:

$$
A_{500}=\bar{A}_{500} \quad 0,031
$$

siendo $A_{500}$ el valor de la absorbancia a $500 \mathrm{~nm}$ obtenido para una concentración dada de monoacilderivado.

Las conclusiones obtenidas del análisis de la varianza realizado con los datos correspondientes al diacilderivado son las mismas que en el caso anterior. El valor de la desviación típica entre replicados en este caso es:

$$
s=0,0274
$$

y los Límites de Confianza (9 grados de libertad y una probabilidad del $95 \%$ ):

$$
A_{510}=\bar{A}_{510} \pm 0,020
$$

donde $A_{510}$ es el valor de la absorbancia a $510 \mathrm{~nm}$ obtenido para una concentración dada de diacilderivado.

\subsection{Eficacia de la preparación de las mues-} tras.

Como ya vimos en el punto 2.5 el procedimiento necesario para preparar las muestras de mono- $y$ diacilderivado en las condiciones requeridas por el método analítico es bastante complicado. Por lo tanto, hemos creído conveniente comprobar si los datos obtenidos por este procedimiento están dentro de los Límites de Confianza establecidos para cada uno de los productos.

Para analizar el error que puede cometerse en la cuantificación debido a la complejidad del método propuesto hemos preparado una serie de muestras, tanto de mono- como de diacilderivado, de concentraciones conocidas. Estas disoluciones han sido analizadas espectrofotométricamente para comprobar previamente su concentración y a continuación se han depositado cantidades conocidas de cada una de ellas sobre placas de gel de sílice y se ha seguido todo el procedimiento descrito con anterioridad.

Los resultados obtenidos corroboran la validez del método propuesto ya que las diferencias encontradas entre las concentraciones de las disoluciones de partida y las de las muestras recuperadas de las placas han sido siempre inferiores a los Límites de Confianza establecidos para cada producto.

\section{3. Aplicaciones del método analítico.}

La puesta a punto de una técnica eficaz para la determinación de acilderivados de arilaminoalcoholes era un obstáculo importante a la hora de estudiar la síntesis de estos productos. La cuantificación de los mismos, necesaria para establecer el rendimiento de la reacción de síntesis en función de las condiciones utilizadas, había sido ensayada, sin éxito, en nuestros laboratorios con anterioridad mediante técnicas̊ como la cromatografía líquida de alta eficacia o la cromatografía gaseosa.

En principio, la utilización de éstas técnicas habría supuesto una simplificación considerable en la preparación de las muestras. Sin embargo, la imposibilidad de poder evaluar los distintos productos formados en la reacción en un solo análisis nos hizo desestimar 
estos procedimientos y recurrir a la espectrofotometría de absorción que nos proporciona unos resultados muy satisfactorios a partir de las soluciones previamente purificadas de cada uno de los productos.

Este método analítico ha establecido, por tanto, un procedimiento útil para la determinación de productos derivados de arilaminoalcoholes, escasamente descritos en la bibliografía y que debido a su naturaleza anfifílica pueden presentar propiedades de cierto interés como agentes superficiales, si bien el estudio de dichas propiedades serán el objetivo de investigaciones posteriores.

\section{BIBLIOGRAFIA}

1. Vázquez Roncero, A.; Maestro Durán, R.; Graciani Constante, E. y Janer del Valle, C.- "Nuevos derivados de la anilina en aceites asociados al "Síndrome Tóxico". I. Esteres grasos del 1,2-propanodiol-3-aminofenilo".- Grasas y Aceites 35 (1984) 15-21.

2. Vázquez Roncero. A.; Maestro Durán, R.; Janer del Valle, C.; Graciani Constante, E. y Gómez Gómez, R.- "Nuevos derivados de la anilina en aceites asociados al "Síndrome Tóxico". II. Síntesis de mono- y diésteres grasos del 3fenilamino-1,2-propanodiol".- Grasas y Aceites 36 (1985) 382-89.

3. Vázquez Roncero, A. y Gómez Gómez, R.- "Esteres grasos de 3-arilamino-1,2-propanodiol.-" Grasas y Aceites 38 (1987) 87-92.

4. Sakai, S.; Suzuki, K.; Mori, H y Fujino, M.- "Als Forbreagens für primäre aromatische Amine.- "Jap. Analyst 9 (1960) 862-64.- Según Z. Anal. Chem. 183 (1961) 450.

5. Albi, M. A. y Vioque, A.- "Determinación espectrofotométrica de anilidas de ácidos grasos y anilina libre en aceites comestibles".- Grasas y Aceites 34 (1982) 24-28.

(Recibido: Octubre 1990). 